\title{
Há diferenças nas práticas de terapia nutricional entre pacientes pediátricos graves clínicos e cirúrgicos?
}

\author{
Are there differences in nutritional therapy practices between clinical and surgical severe pe- \\ diatric patients?
}

DOI: $10.37111 /$ braspenj.2019344010

Daniela B. Hauschild'

Julia C. Ventura

Luna D. A. Oliveira

Taís T. Silveira²

Eliana Barbosa ${ }^{3}$

Nilzete Liberato ${ }^{4}$

Yara M. F. Moreno

\begin{abstract}
RESUMO
Introdução: Pacientes pediátricos graves admitidos por motivos cirúrgicos podem diferir de pacientes clínicos. Entretanto, diretrizes destinadas à terapia nutricional (TN) incluem pacientes clínicos e cirúrgicos. O objetivo deste estudo foi descrever as práticas de TN de pacientes pediátricos graves admitidos por motivos clínicos e cirúrgicos. Método: Coorte prospectiva com pacientes pediátricos graves, entre 1 mês e 15 anos de idade, admitidos em unidade de terapia intensiva. Pacientes que receberam TN via oral, tiveram alta $<48$ horas ou foram a óbito nas primeiras 72 horas foram excluídos. Foram coletados dados clínicos e demográficos. $O$ estado nutricional foi avaliado na admissão e de TN dos primeiros 7 dias. Testes de Qui-quadrado e Mann-Whitney foram aplicados e $\mathrm{p}<0,05$ considerado significativo. Resultados: Foram incluídos 201 pacientes, com idade mediana de 2,2 anos, $154(76,6 \%)$ admitidos por motivos clínicos e $47(23,4 \%)$ por motivos cirúrgicos. Comparados aos pacientes clínicos, pacientes cirúrgicos apresentaram maior mediana de idade $(5,0$ vs. 1,4 anos; $p=0,035)$, menor Pediatric Index of Mortality $2(1,1$ vs. $6,1 \% ; p<0,001)$, maior prevalência de doenças crônicas complexas (17 vs. $47 \% ; p<0,001)$. Não houve diferença quanto ao estado nutricional na admissão. Pacientes cirúrgicos apresentaram maior tempo para início da TN $(22,3$ vs. $16,3 \mathrm{~h} ; \mathrm{p}=0,016)$, maior prevalência de nutrição parenteral $(31,6$ vs. $15,1 \% ; p=0,019)$, menor oferta de energia $(24,5$ vs. $35,9 \mathrm{kcal} / \mathrm{kg} / \mathrm{d} ; p=0,003)$ e proteína $(0,82$ vs. $0,99 \mathrm{~g} / \mathrm{kg} / \mathrm{d} ; \mathrm{p}=0,026)$ e maior prevalência de hipoalimentação $(82,6 \mathrm{vs.} 50 \%$; $p<0,001)$. Observou-se maior prevalência de distensão abdominal $(36,2$ vs. $21,4 \% ; p=0,04)$ e constipação $(38,3$ vs. $16,9 \% ; p=0,002)$ nos pacientes cirúrgicos. Conclusões: As práticas de TN diferem entre pacientes clínicos e cirúrgicos. Pacientes cirúrgicos apresentaram maior idade, menor escore de gravidade, demoraram mais para iniciar TN, e apresentaram maior prevalência de hipoalimentação e de distensão abdominal. Desta maneira, sugere-se que os protocolos de TN devam ser individualizados, conforme motivo de internação.
\end{abstract}

\section{ABSTRACT}

Introduction: Pediatric critically ill patients admitted for surgical reasons may differ from medical patients. However, guidelines for nutritional therapy (NT) include both medical and surgical patients. The aim of this study was to describe the NT practices of critically ill children admitted for medical and surgical reasons. Methods: Prospective cohort study conducted with critically ill children, between 1 month and 15 years old, admitted in a pediatric intensive care unit. Patients who were discharged within the first 48 hours, died within the first 72 hours or who received oral NT were excluded. Clinical and demographic were collected. Nutritional status was assessed at admission and NT data from the first 7 days was collected. Chi-square and Mann-Whitney tests were applied and $p<0.05$ was considered significant. Results: A total of 201 patients were included, with a median age of 2.2 years, 154 (76.6\%) were admitted for medical reasons and $47(23.4 \%)$ for surgical reasons. Compared to medical patients, surgical patients had a higher median age (5.0 vs. 1.4 years; $p=0.035)$, lower Pediatric Index of Mortality $2(1.1$ vs. $6.1 \% ; p<0.001)$ and higher prevalence of complex chronic diseases (17 vs. $47 \% ; p<0.001)$. There was no difference regarding nutritional status. Surgical patients showed higher median time for NT initiation $(22.3$ vs. $16.3 \mathrm{~h} ; \mathrm{p}=0.016)$, higher prevalence of parenteral nutrition (31.6 vs. $15.1 \% ; p=0.019)$, lower energy $(24.5 \mathrm{vs}$. $35.9 \mathrm{kcal} / \mathrm{kg} / \mathrm{d} ; \mathrm{p}=0.003)$ and protein $(0.82 \mathrm{vs} .0 .99 \mathrm{~g} / \mathrm{kg} / \mathrm{d} ; \mathrm{p}=0.026)$ intake and higher prevalence of underfeeding $(82.6 \mathrm{vs.} 50 \% ; p<0.001)$. There was a higher prevalence of abdominal distension ( 36.2 vs. $21.4 \% ; p=0.04)$ and constipation $(38.3$ vs. $16.9 \% ; p=0.002)$ in surgical patients. Conclusion: Surgical patients were older, less severe, had longer time for NT initiation, higher prevalence of underfeeding and abdominal distension in the first 7 days. NT protocols should be individualized according to the reason for hospitalization.
Aceito para publicação

11 de novembro de 2019

1. Doutora, Departamento de Nutrição, Universidade Federal de Santa Catarina, Florianópolis, SC, Brasil. Mestre, Programa de Pós-Graduação em Nutrição, Universidade Federal de Santa Catarina, Florianópolis, SC, Brasil. Mestre em Nutrição, Hospital Infantil Joana de Gusmão, Florianópolis, SC, Brasil.

2. Mestre, Unidade de Terapia Intensiva Pediátrica, Hospital Infantil Joana de Gusmão, Florianópolis, SC, Brasil.

3. Doutora, Programa de Pós-Graduação em Nutrição e Departamento de Nutrição, Úniversidade Federal de Santa Catarina, Florianópolis, SC, Brasil. 


\section{INTRODUÇÃO}

Em pacientes pediátricos graves, após o surgimento de evento como processo cirúrgico, trauma ou sepse, observa-se a resposta metabólica ao estresse como parte da resposta adaptativa à situação crítica, a qual é desencadeada com objetivo de aumentar o fornecimento de substratos de energia para os tecidos vitais. Como consequência, destaca-se o catabolismo

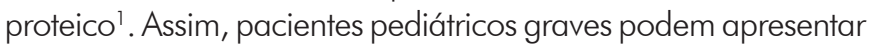
deterioração do estado nutricional durante a internação².

Pacientes podem ser admitidos em unidade de terapia intensiva pediátrica (UTIP) por motivos cirúrgicos (objetivo da admissão na UTIP para cuidados de pós-operatório ou após trauma); ou por motivos clínicos (objetivo da admissão na UTIP por doença sem intervenção cirúrgica antes da admissão) ${ }^{3}$. Pacientes cirúrgicos pediátricos respondem ao estresse metabólico de maneira diferenciada ${ }^{4}$. Usualmente, apresentam-se menos graves na admissão, com consequente menor tempo de internação em unidade de terapia intensiva $(\mathrm{UTI})^{5}$. Manter o estado nutricional desses pacientes é fundamental, não apenas para auxiliar na cicatrização, mas também para minimizar o catabolismo proteico ${ }^{4}$. A extensão do catabolismo está relacionada à magnitude do estresse cirúrgico, e a deterioração do estado nutricional é um fator de risco para complicações pós-operatórias ${ }^{6}$.

Entretanto, a maioria das diretrizes destinadas à terapia nutricional do paciente pediátrico grave é baseada em UTIPs heterogêneas, incluindo pacientes clínicos e cirúrgicos ${ }^{7}$. Definir as necessidades nutricionais de pacientes pediátricos graves ainda é um desafio e estas podem ser diferenciadas conforme a condição clínica do paciente admitido na UTI 1,8 . Os déficits de energia e de proteína estão associados com aumento na morbimortalidade nessa população. Dessa forma, tanto a ingestão de energia quanto a de proteína tornam-se necessárias para minimizar a perda de massa magra, ${ }^{9} 10$.

A literatura ainda é escassa a respeito das diferenças nas práticas de terapia nutricional existentes entre pacientes clínicos e cirúrgicos no ambiente de terapia intensiva pediátrica. Nesse contexto, o objetivo do presente estudo foi descrever as práticas de terapia nutricional de pacientes pediátricos graves admitidos por motivos clínicos e cirúrgicos.

\section{MÉTODO}

\section{Desenho e População do Estudo}

Estudo prospectivo, observacional, longitudinal, realizado em um hospital terciário do sul do Brasil, entre julho de 2013 e janeiro de 2016. O recrutamento foi realizado nas primeiras 48 horas de admissão na UTIP e foram incluídos pacientes entre 1 mês e 15 anos de idade, de ambos os sexos. Foram excluídos pacientes que receberam alta nas primeiras 48 horas, foram a óbito nas primeiras 72 horas de internação, impossibilidade de realizar avaliação nutricional em 72 horas, ou iniciaram a terapia nutricional com dieta via oral. Em caso de readmissão na UTIP, o paciente foi incluído somente na primeira internação. $\bigcirc$ estudo foi aprovado pelo Comitê de Ética em Pesquisa em Humanos da Universidade
(\# 402.469). O consentimento informado por escrito foi obtido dos pais ou responsáveis legais de todos os pacientes incluídos no estudo.

\section{Variáveis Clínicas}

Características demográficas e clínicas foram coletadas a partir do prontuário. $O$ diagnóstico primário foi categorizado em motivo cirúrgico ou clínico. Como índice prognóstico de mortalidade, foi utilizado o Pediatric Index of Mortality 2 (PIM 2), expresso em percentual ${ }^{11}$. A presença de doenças crônicas complexas (DCC) foi documentada ${ }^{12}$. Foram avaliados os desfechos clínicos de infecção nosocomial, tempo em ventilação mecânica (VM), tempo de permanência hospitalar e na UTIP, e mortalidade. A presença de infecção nosocomial foi definida como a presença de pneumonia adquirida, infecção do trato urinário e infecções sanguíneas, desenvolvidas após 48 horas de admissão na UTIP.

\section{Avaliação Antropométrica e Laboratorial}

Parâmetros antropométricos e laboratoriais foram avaliados em até 72 horas após admissão na UTIP. A avaliação antropométrica foi realizada por profissional previamente treinado e incluiu dados de peso corporal, comprimento/estatura e circunferência do braço $(C B)^{13}$.

O peso corporal foi avaliado por meio de balança pediátrica com precisão de 0,05 kg (Filizola BP Baby ${ }^{\circledR}$, São Paulo, Brasil). O comprimento/estatura foi aferido com antropômetro pediátrico com escala de precisão de $0,1 \mathrm{~cm}$ (Caumaq $^{\circledR}$, Cachoeira do Sul, Brasil) ou fita métrica inelástica, flexível, graduada em cm, e os valores expressos em $0,1 \mathrm{~cm}$ (TBW ${ }^{\circledR}$, São Paulo, Brasil). Na impossibilidade de aferição do comprimento/estatura, foi realizada a estimativa por meio da aferição da altura do joelho ${ }^{14}$. $\mathrm{O}$ indicador de índice de massa corporal-para-idade (IMC/I), expresso em escore-z, foi calculado com auxilio do software World Health Organization (WHO) Anthro ou WHO AnthroPlus ${ }^{\circledR}$ (Geneva, Suíça).

A CB foi avaliada com fita métrica inelástica, flexível, graduada em $\mathrm{cm}$, e os valores expressos em $0,1 \mathrm{~cm}$. $O$ escore-z de CB-para-idade (CB/I) foi calculado de acordo com os valores da WHO, em crianças menores de 5 anos, ou de acordo com Frisancho ${ }^{15}$, em crianças maiores de 5 anos.

A albumina sérica foi avaliada pelo método verde de bromocresol, por meio do Kit Quimialb - Albumina e equipamento automatizado QUIMISAT 450 (EBRAM ${ }^{\circledR}$ Produtos Laboratoriais Ltda., São Paulo, Brasil). Os valores foram expressos em $\mathrm{g} / \mathrm{dL}$. A proteína $\mathrm{C}$-reativa (PCR) sérica foi mensurada pelo método de imunoturbidimetria Látex por meio do Kit Turb - PCR e equipamento automatizado QUIMISAT 450 (EBRAM ${ }^{\circledast}$ Produtos Laboratoriais Ltda., São Paulo, Brasil). Os valores foram expressos em $\mathrm{mg} / \mathrm{L}$.

\section{Práticas de Terapia Nutricional}

Foram coletados os dados de terapia nutricional dos primeiros 7 dias de admissão na UTIP referentes ao tempo 
para início da dieta (em horas), via de administração (enteral, enteral + parenteral ou parenteral), prescrição e infusão de energia e proteína, e tempo para atingir a meta de energia e proteína. Terapia nutricional precoce, enteral e/ou parenteral, foi considerada como início nas primeiras 24 horas após admissão na UTIP. A necessidade energética foi calculada pela equação de Schofield ${ }^{16}$, sem adição de fator injúria. A necessidade proteica mínima foi de 1,5 g/ $\mathrm{kg} / \mathrm{dia}^{17}$. A adequação de energia e proteína foi calculada como o percentual da necessidade pela ingestão atual, sendo <80\% como hipoalimentação e > 120\% hiperalimentação.

Foram coletados o número, motivo e tempo (em horas) das interrupções de infusão de dieta enteral; a frequência, consistência e quantidade de fezes diárias; e a presença diária de distensão abdominal dos primeiros 7 dias de admissão na UTIP.

\section{Análise Estatística}

A análise de dados estatísticos foi realizada no software STATA 11.0 (Stata Corporation ${ }^{\circledR}$, Texas, EUA). As variáveis categóricas foram descritas em valores absolutos e frequência. As variáveis quantitativas foram descritas como mediana e intervalo interquartil (IQR). Para comparação das características clínicas, de estado nutricional e de terapia nutricional entre os pacientes clínicos e cirúrgicos, foram utilizados os testes de Mann-Whitney, Qui-quadrado ou Qui-quadrado de Fisher. Para todas as análises, $p<0,05$ foi considerado significativo.

\section{RESULTADOS}

\section{Caracterização da População}

Dentre os 715 pacientes pediátricos graves que foram internados na UTIP, 76 não preencheram os critérios de inclusão e 427 preencheram os critérios de exclusão. Entre os 212 pacientes recrutados, 201 foram incluídos no estudo (Figura 1). A idade mediana foi de 2,2 anos (IQR 0,45; 7,44), $62,7 \%$ do sexo masculino, 154 (76,6\%) foram admitidos por motivos clínicos e 47 (23,4\%) por motivos cirúrgicos. Dentre os pacientes admitidos por motivos cirúrgicos, 59,6\% foram por cirurgia abdominal, 21 ,3\% cardíaca, 10,6\% neurológica e $8,5 \%$ ortopédica.

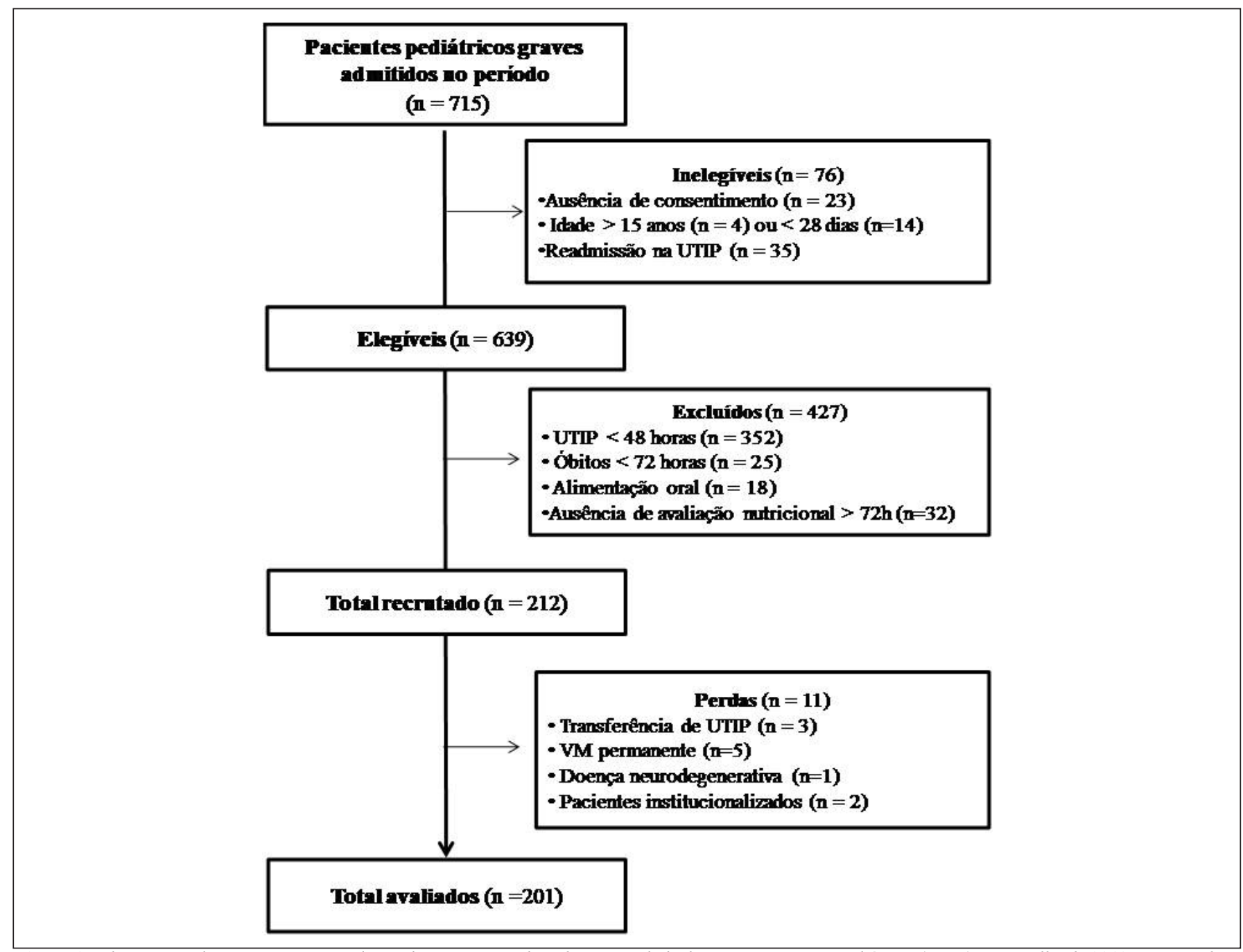

Figura 1 - Fluxograma de recrutamento e inclusão dos pacientes admitidos em unidade de terapia intensiva pediátrica (UTIP), entre julho de 2013 e janeiro de 2016. VM: ventilação mecânica. 


\section{Variáveis Clínicas}

Em comparação com os pacientes clínicos, pacientes cirúrgicos apresentaram maior mediana de idade $(p=0,035)$, menor PIM 2 ( $<<0,001)$, e maior prevalência de DCC $(p<0,001)$. Não foram observadas diferenças no tempo de internação hospitalar e na UTIP, entretanto, o tempo em VM foi menor em pacientes cirúrgicos $(p=0,016)$. A taxa de mortalidade geral foi de 12,9\%, não havendo diferença entre os grupos (Tabela 1).

Tabela 1 - Caracterização de pacientes pediátricos graves estratificados pelo motivo de admissão em uma unidade de terapia intensiva pediátrica, entre julho de 2013 e janeiro de $2016(n=210)$.

\begin{tabular}{|c|c|c|c|}
\hline \multirow{2}{*}{ Variáveis } & Clínicos (n=154) & Cirúrgicos (n=47) & \multirow[b]{2}{*}{ p-valor } \\
\hline & $\begin{array}{c}\text { Mediana [IQR]/ } \\
\mathrm{n}(\%) \\
\end{array}$ & $\begin{array}{c}\text { Mediana [IQR]/ } \\
\mathrm{n}(\%) \\
\end{array}$ & \\
\hline Sexo (masculino) & $97(63,0)$ & $29(61,7)$ & 0,873 \\
\hline Idade (anos) & $1,42(0,32 ; 7,05)$ & $4,98(1,02 ; 8,26)$ & 0,035 \\
\hline PIM 2 (\%) & $6,1(1,6 ; 21,0)$ & $1,1(0,6 ; 2,9)$ & $<0,001$ \\
\hline \multicolumn{4}{|l|}{ Parâmetros antropométricos na admissão } \\
\hline z-IMC/I & $-0,02(-1,14 ; 0,97)$ & $-0,49(-2,52 ; 0,73)$ & 0,072 \\
\hline$z-C B / l$ & $0,09(-1,24 ; 1,02)$ & $-0,19(-1,15 ; 0,66)$ & 0,463 \\
\hline \multicolumn{4}{|l|}{ Parâmetros laboratoriais na admissão } \\
\hline Albumina $(g / d L)(n=190)$ & $3,0(2,5 ; 3,5)$ & $2,9(2,5 ; 3,3)$ & 0,183 \\
\hline \multicolumn{4}{|l|}{ Via } \\
\hline Enteral exclusiva & $124(84,9)$ & $26(68,4)$ & 0,019 \\
\hline Parenteral exclusiva ou suplementar & $22(15,1)$ & $12(31,6)$ & \\
\hline Início precoce (24h) & $105(69,5)$ & $25(53,2)$ & 0,039 \\
\hline \multicolumn{4}{|l|}{ Energia } \\
\hline Ingestão em 7 dias (kcal/kg/dia) & $35,9(20,7 ; 50,4)$ & $24,5(12,6 ; 33,2)$ & 0,003 \\
\hline Atingir a meta em 4 dias & $93(60,4)$ & $16(34,0)$ & 0,002 \\
\hline \multicolumn{4}{|l|}{ Adequação } \\
\hline Adequado (80-120\%) & $49(32,7)$ & $4(8,7)$ & \\
\hline Distensão abdominal & $33(21,4)$ & $17(36,2)$ & 0,041 \\
\hline Constipação & $26(16,9)$ & $18(38,3)$ & 0,002 \\
\hline Diarreia & $27(17,5)$ & $3(6,4)$ & 0,060 \\
\hline Interrupção da enteral & $106(68,8)$ & $23(48,9)$ & 0,015 \\
\hline Número de interrupções da enteral & $2(0 ; 6)$ & $0(0 ; 5)$ & 0,011 \\
\hline \multicolumn{4}{|l|}{ Desfechos clínicos } \\
\hline Uso de VM & $125(81,2)$ & $37(23,4)$ & 0,679 \\
\hline Duração de VM (dias) (n=162) & $6(3 ; 11)$ & $3(2 ; 8)$ & 0,016 \\
\hline Infecção nosocomial & $44(29,5)$ & $7(15,2)$ & 0,054 \\
\hline Tempo de internação UTIP (dias) & $7(5 ; 13)$ & $7(3 ; 10)$ & 0,708 \\
\hline Tempo de internação hospitalar (dias) & $23(14 ; 45)$ & $21(11 ; 30)$ & 0,133 \\
\hline Mortalidade & $19(12,3)$ & $7(14,9)$ & 0,648 \\
\hline
\end{tabular}




\section{Avaliação Antropométrica e Laboratorial}

Não houve diferença entre pacientes cirúrgicos e clínicos quanto ao escore-z de IMC/I $(p=0,072)$ e $C B / I(p=0,463)$. As concentrações de PCR e albumina não foram diferentes entre os grupos (Tabela 1).

\section{Práticas de Terapia Nutricional}

Pacientes cirúrgicos apresentaram maior tempo para o início da terapia nutricional $(p=0,016)$, sendo a prevalência de terapia nutricional precoce menor nesse grupo $(p=0,039)$. A prevalência de nutrição parenteral foi maior em pacientes cirúrgicos $(p=0,019)$. Houve menor ingestão de energia $(p=0,003)$ e proteína $(p=0,026)$ e maior prevalência de hipoalimentação $(p<0,001)$ nos pacientes cirúrgicos. Foi observado que $34 \%$ dos pacientes cirúrgicos e $60,4 \%$ dos pacientes clínicos atingiram a meta energética em até 4 dias $(p=0,002)$ (Tabela 1).

Foi observada maior prevalência de distensão abdominal $(p=0,04)$ e constipação $(p=0,002)$ nos pacientes cirúrgicos, entretanto, a prevalência e o número de interrupções da terapia nutricional enteral foram menores nos pacientes cirúrgicos. Não foi observada diferença entre grupos na ocorrência de diarreia (Tabela 1).

\section{DISCUSSÃO}

No presente estudo, conduzido em uma UTIP mista, foi observada prevalência de $23,4 \%$ de admissão por motivo cirúrgico. Pacientes admitidos por motivos cirúrgicos apresentaram maior idade e menor gravidade. As práticas de terapia nutricional foram diferenciadas entre os pacientes clínicos e cirúrgicos - houve menor prevalência de início precoce da terapia nutricional, maior prevalência de hipoalimentação e menor ingestão de energia e proteína em 7 dias nos pacientes cirúrgicos. Esses dados reforçam a importância de protocolos específicos de terapia nutricional, de acordo com o motivo de internação na UTIP.

Foram evidenciadas diferenças entre pacientes clínicos e cirúrgicos em relação à idade e à gravidade. Achado similar foi observado em estudo com 802 pacientes admitidos em uma UTIP, com idade média de 8 anos, e prevalência de $55 \%$ de admissão por motivos cirúrgicos. Foi observado que pacientes cirúrgicos apresentaram menor gravidade, avaliado pelo Pediatric Risk of Mortality III (PRISM III), menor tempo de internação e menor custo de internação, quando comparados aos pacientes clínicos ${ }^{18}$. Em estudo com 130 pacientes pediátricos graves, aqueles admitidos por motivos clínicos ou cardiopatia congênita apresentaram menor idade comparada aos pacientes cirúrgicos, corroborando com os nossos achados ${ }^{19}$. A literatura aponta diversos benefícios da nutrição enteral, incluindo redução das taxas de infecção e manutenção da integridade da mucosa intestinal. Ainda, preconiza-se o início precoce da terapia nutricional, em até
24 a 48 horas após a admissão. Em adultos, hemodinamicamente estáveis, as principais diretrizes recomendam o início da nutrição enteral dentro de 6 horas após a admissão na UTI, de forma segura, tanto em pacientes clínicos quanto cirúrgicos. Essa recomendação deve ser considerada também na população pediátrica ${ }^{17,20,21}$.

Entretanto, no presente estudo, pacientes cirúrgicos iniciaram a terapia nutricional mais tardiamente, com menor prevalência de terapia nutricional precoce $(53,2 \%)$. Outros estudos também demonstraram que pacientes cirúrgicos demoram mais para iniciar a terapia nutricional ${ }^{19,22}$. Uma das possíveis causas é o fato dos cirurgiões e intensivistas optarem por adiar o início da terapia nutricional devido a diferentes razões, como a possibilidade de retornar à sala de cirurgia ou de extubação 22 .

Em estudo internacional, realizado em 269 UTIs, com 5.497 pacientes adultos em VM, 37,7\% apresentaram diagnóstico cirúrgico na admissão. Foi observado que pacientes cirúrgicos apresentaram menor probabilidade de receber nutrição enteral $(54,6 \%$ vs. $77,8 \%)$ e maior probabilidade de receber nutrição parenteral (13,9\% vs. 4,4\%). Ainda, pacientes cirúrgicos receberam aporte energético menor, quando comparado aos pacientes clínicos ${ }^{22}$.

A Sociedade Americana de Nutrição Parenteral e Enteral (ASPEN) recomenda valores superiores de ingestão diária de proteínas em pacientes pediátricos graves, quando comparados aos valores recomendados para crianças saudáveis, sendo o mínimo recomendado de 1,5 g/ $/ \mathrm{kg} / \mathrm{dia}^{17}$. Em nosso estudo, pacientes cirúrgicos receberam menor aporte de energia e de proteína nos primeiros 7 dias de internação, com mediana de ingestão proteica de 0,82 g/ $\mathrm{kg} /$ dia, abaixo do mínimo recomendado. A associação entre adequação da ingestão de energia e menor mortalidade em 60 dias já foi descrita $^{10}$. Em trabalho prospectivo, multicêntrico, que incluiu 59 UTIPs de 15 países, com 1.245 pacientes pediátricos graves entre um mês e 18 anos, em VM por mais de 48 horas, aproximadamente $77 \%$ receberam terapia nutricional enteral. A ingestão de proteína, por meio da nutrição enteral, superior a $60 \%$ do prescrito, esteve associada à menor mortalidade em 60 dias com odds ratio (OR) de 0,1 4 (intervalo de confiança [IC] 95\% 0,04-0,52; $p=0,003)$, em comparação àqueles que receberam menos de $20 \%$ do prescrito?.

Entre as causas para a baixa ingestão de energia e proteína estão as barreiras à terapia nutricional, que podem resultar em ingestão energética e de proteína inadequada, sendo o tempo prolongado para o início da terapia nutricional e as frequentes interrupções fatores que contribuem para a baixa ingestão de energia e proteína ${ }^{21}$.

Em coorte com 519 pacientes pediátricos graves cirúrgicos, $66 \%$ receberam nutrição enteral, com mediana de 2 dias para o início da terapia nutricional. Atrasos 
ao iniciar a terapia nutricional e a duração prolongada das interrupções foram associados à falha em atingir as necessidades proteicas de pacientes pediátricos graves ${ }^{21}$. De forma similar, estudo com 100 pacientes admitidos em UTIP, dos quais $62 \%$ foram admitidos por motivos cirúrgicos, pacientes com menor idade ( 0 a 6 meses) apresentaram maior número de interrupções e por período mais prolongado comparados a pacientes com maior idade (acima de 6 meses). As interrupções para extubação ou procedimento cirúrgico foram as causas que acarretaram maior tempo de dieta interrompida ${ }^{23}$.

Vários estudos demonstraram que a implementação de protocolo de terapia nutricional pode reduzir significativamente o tempo para o início da nutrição enteral e tempo para atingir as metas de energia e proteína. Mais estudos são necessários para desenvolver e examinar o papel dos algoritmos na otimização da oferta de nutrientes aos pacientes cirúrgicos na UTIP17,21,22,24.

Nosso estudo apresenta limitações, incluindo a realização em uma única instituição e o pequeno tamanho amostral. Entretanto, o recrutamento ocorreu em um centro de referência do sul do Brasil. A avaliação de medidas antropométricas em UTIP ainda é controversa. Contudo, dados de peso corporal e CB são considerados medidas relevantes para avaliação do estado nutricional. A determinação das necessidades energéticas ocorreu por meio da estimativa baseada em fórmulas preditoras, o que pode acarretar hipo ou hiperalimentação. No entanto, a equação utilizada é a preconizada pela diretriz da ASPEN ${ }^{17}$.

\section{CONCLUSÃO}

Pacientes cirúrgicos apresentaram maior idade, menor escore de gravidade, demoraram mais para iniciar a terapia nutricional, e apresentaram maior prevalência de hipoalimentação e de distensão abdominal nos primeiros 7 dias de internação na UTIP. As razões para as diferenças nas características de pacientes cirúrgicos comparados aos clínicos requerem maiores esclarecimentos e podem ter implicações tanto na utilização de recursos quanto no custo, bem como na evolução clínica. A literatura demonstra que o início precoce da terapia nutricional pode melhorar o aporte de energia e proteína, e a implementação de protocolos pode facilitar esse processo. Assim, protocolos de terapia nutricional devem ser individualizados, conforme o motivo de internação.

\section{AGRADECIMENTOS}

Agradecemos à equipe do Hospital Infantil Joana de Gusmão, por seu apoio durante este estudo, e às crianças e seus pais, que concordaram em participar deste estudo.

\section{REFERÊNCIAS}

1. Joosten KFM, KerklaanD, Verbruggen SCAT. Nutritional support and the role of the stress response in critically ill children. Vol. 19. Current opinion in clinical nutrition and metabolic care. Philadelphia: Lippincott Williams and Wilkins; 2016. p.226-33.

2. Hulst JM, van Goudoever JB, Zimmermann LJI, Hop WCJ, Albers MJIJ, Tibboel D, et al. The effect of cumulative energy and protein deficiency on anthropometric parameters in a pediatric ICU population. Clin Nutr. 2004;23(6):1381-9.

3. Joffe A HL. Cochrane Database of Systematic Reviews Nutritional support for critically ill children (Review). Cochrane Database Syst Rev [Internet]. 2016 [cited 2019 Oct 7]; Available from: www.cochranelibrary.com

4. Herman R, Btaiche I, Teitelbaum DH. Nutrition support in the pediatric surgical patient. SurgClinNorthAm.2011;91(3):511-41.

5. Nathanson BH, McGee WT, Lederman E, Higgins TL. Differences in severity and resource utilization for medical and surgical ICU patients. Crit Care. 2013;17(Suppl 2):P490.

6. Weimann A, Braga M, Carli F, Higashiguchi T, Hübner M, Klek $\mathrm{S}$, et al. ESPEN guideline: Clinical nutrition in surgery. Clin Nutr. 2017;36(3):623-50.

7. Tume LN, Valla FV, Floh AA, Goday P, Chaparro CJ, Larsen B, et al. Priorities for nutrition research in pediatric critical care. JPEN J Parenter Enteral Nutr. 2018;43(7):853-62.

8. Joosten K, Van Puffelen E, Verbruggen S. Optimal nutrition in the paediatric ICU. Vol. 19. Current opinion in clinical nutrition and metabolic care. Philadelphia: Lippincott Williams and Wilkins; 2016. p.131-7.

9. Mehta NM, Bechard LJ, Zurakowski D, Duggan CP, Heyland DK. Adequate enteral protein intake is inversely associated with 60-d mortality in critically ill children: a multicenter, prospective, cohort study. Am J Clin Nutr. 2015;102(1):199-206.

10. Mehta NM, Bechard LJ, Cahill N, Wang M, Day A, Duggan $\mathrm{CP}$, et al. Nutritional practices and their relationship to clinical outcomes in critically ill children: an international multicenter cohort study. Crit Care Med. 2012;40(7):2204-11.

11. Slater A, Shann F, Pearson G. PIM2: a revised version of the Paediatric Index of Mortality. Intensive Care Med. 2003;29(2):278-85.

12. Edwards JD, Houtrow AJ, Vasilevskis EE, Rehm RS, Markovitz BP, Graham RJ, et al. Chronic conditions among children admitted to U.S. pediatric intensive care units. Crit Care Med. 2012;40(7):2196-203.

13. World Health Organization. Physical status: the use and interpretation of anthropometry: Report of a WHO Expert Committee. Geneva: World Health Organization; 1995.

14. Chumlea WC, Guo SS, Steinbaugh ML. Prediction of stature from knee height for black and white adults and children with application to mobility-impaired or handicapped persons. J Am Diet Assoc. 1194;94(12):1385-8.

15. Frisancho AR. New norms of upper limb fat and muscle areas for assessment of nutritional status. Am J Clin Nutr. 1981;34(11):2540-5.

16. Schofield W. Predicting basal metabolic rate, new standards and review of previous work. Hum Nutr Clin Nutr. 1985;39(Suppl 1):5-41.

17. Mehta NM, Skillman HE, Irving SY, Coss-Bu JA, Vermilyea S, Farrington EA, et al. Guidelines for the provision and assessment of nutrition support therapy in the pediatric critically ill patient: Society of Critical Care Medicine and American Society for Parenteral and Enteral Nutrition. J Parenter Enter Nutr. 2017;41(5):706-42.

18. Hsu BS, Brazelton TB. A comparison of costs between medical and surgical patients in an academic pediatric intensive care unit. Wis Med J. 2015;114(6):236-9.

19. Mara J, Gentles E, Alfheeaid HA, Diamantidi K, Spenceley N, Davidson $\mathrm{M}$, et al. An evaluation of enteral nutrition practices 
and nutritional provision in children during the entire length of stay in critical care. BMC Pediatr. 2014;14:186.

20. McClave SA, Taylor BE, Martindale RG, Warren MM, Johnson $\mathrm{DR}$, Braunschweig $\mathrm{C}$, et al. Guidelines for the provision and assessment of nutrition support therapy in the adult critically ill patient: Society of Critical Care Medicine (SCCM) and American Society for Parenteral and Enteral Nutrition (A.S.P.E.N.). JPEN J Parenter Enteral Nutr. 2016;40(2):159-211.

21. Velazco CS, Zurakowski D, Fullerton BS, Bechard LJ, Jaksic T, Mehta NM. Nutrient delivery in mechanically ventilated surgical patients in the pediatric critical care unit. J Pediatr Surg. 2017;52(1):145-8.
22. Drover JW, Cahill NE, Kutsogiannis J, Pagliarello G, Wischmeyer P, Wang M, et al. Nutrition therapy for the critically ill surgical patient: we need to do better! J Parenter Enter Nutr. 2010;34(6):644-52.

23. Keehn A, O'Brien C, Mazurak V, Brunet-Wood K, Joffe A, De Caen A, et al. Epidemiology of interruptions to nutrition support in critically Ill children in the pediatric intensive care unit. J Parenter Enter Nutr. 2015;39(2):211-7.

24. Hamilton S, McAleer DM, Ariagno K, Barrett M, Stenquist N, Duggan CP, et al. A stepwise enteral nutrition algorithm for critically ill children helps achieve nutrient delivery goals. Pediatr Crit Care Med. 2014;15(7):583-9.

Local de realização do estudo: Universidade Federal de Santa Catarina - Departamento de Nutrição e Hospital Infantil Joana de Gusmão - Florianópolis, SC, Brasil.

Conflito de interesse: As autoras declaram não haver.

Financiamento: Hauschild DB, Ventura JC, Oliveira LDA e Silveira TT receberam bolsa de estudos fornecida pela Coordenação de Aperfeiçoamento de Pessoal de Nível Superior (CAPES).

Foi apresentado como trabalho de destaque no XXIII Congresso Brasileiro de Nutrição Parenteral e Enteral, entre 20 a 23 de outubro de 2019, em Foz do Iguaçu-PR. 